\title{
Calibrating Car Following Parameters for Snowy Road Conditions in the Microscopic Traffic Simulator VISSIM
}

\author{
Johannes Asamer \\ Mobility Department, Austrian Institute of Technology \\ Giefinggasse 2, 1210 Vienna, Austria \\ E-mail: johannes.asamer@ait.ac.at \\ Henk J. van Zuylen \\ Transport and Planning, Delft University of Technology \\ P.O. Box 5048, 2600 GA Delft, The Netherlands \\ E-mail: h.j.vanzuylen@tudelft.nl \\ Bernhard Heilmann \\ Mobility Department, Austrian Institute of Technology \\ Giefinggasse 2, 1210 Vienna, Austria \\ E-mail: bernhard.heilmann@ait.ac.at
}

\begin{abstract}
Calibrated simulation models taking into account adverse weather conditions can be used to optimise traffic management strategies, such as speed adaptation and signal timing optimisation. On snowy road conditions, car following behaviour changes because drivers tend to accelerate more slowly, increase their following distance and drive with lower speeds. Such changes results in lower saturation flow rates at intersections. In order to simulate traffic on snowy road conditions in a valid way, the parameters of a simulation have to be calibrated for adverse weather. This paper identifies the parameters of the car following model in the microscopic simulator VISSIM that are sensitive to snowy road conditions and indicates valid parameter subspaces leading to a good match of simulation model output with observed saturation flow rates and start up delays. In combination with green time of a signalised intersection, saturation flow rate is essential for correctly estimating road capacity.
\end{abstract}




\section{Introduction}

Adverse weather conditions are known to influence strategic, tactical and operational behaviour of traffic participants (e.g. [1], [2], [3]). Although adverse weather influences operational behaviour as e.g. car following, research on this issue is rather limited ([2],[4]). Reduced visibility and slippery road surfaces caused by precipitation lead to lower velocities (see [5], [6]). On the other hand, driving safety hazards brought by slippery or icy roads can motivate drivers to change to other modes of transport in order to avoid getting involved in a car accident (see [1]). Different effects on driving behaviour in the context of calibrating microscopic traffic simulation have been investigated by Sterzin in [4].

Microscopic traffic models reproduce real world traffic dynamics by calculating the movement of vehicles subject to a number of factors such as driver's behaviour, interaction with other vehicles, environmental conditions, network characteristics and control devices as e.g. traffic signals. With the help of microscopic traffic simulators traffic management strategies can be prepared or evaluated. Important examples are the impact of Variable Message Signs on speed adaptation of drivers and signal timing optimisation and its impact on traffic performance and traffic-related emissions (see e.g. [7], [8]). Thus microscopic traffic simulators like VISSIM are important tools for improving existing and introducing new Intelligent Transportation Systems (ITS). In order to obtain reliable results from the microscopic simulation, it is vital that the model accurately captures the driver's real behaviour. To achieve this, the model parameters have to be calibrated for specific conditions.

In this paper we propose a method to modify the parameters of an already well-calibrated model to adverse weather conditions. The main focus lies on adapting the traffic model to snowy road conditions (that can regularly be encountered in Austria and other mountainous countries during wintertime) without taking into account intensity of snowfall. Saturation flow rate and start up delay have been measured on the through lanes of signalised urban intersections in a previous investigation described in [9]. On right and left turn lanes, saturation flow rates are lower compared to through lanes ([10], [11]). Reductions for turning lanes can be taken into account by factors (lower 1) for multiplying saturation flow of through lanes. For all investigations presented in this work, we have used the microscopic traffic simulation model VISSIM. 


\section{Methods}

Calibration of a traffic model is the task of finding optimal parameters to match historical data. This optimisation task involves comparing the model predictions to real-world observations (target values) and minimising the prediction error $([4])$. In this work the target values are saturation flow rate on the through lane (vehicles per hour green per lane - vphgpl) and start up delay, observed during snowy road conditions as reported in [9] (see left column of Table 1 and 2). In that study all three investigated intersections were located on major arterial roads in Vienna with two or three lanes, dedicated only to through traffic (no mixed lanes). The cycle length of the intersection as well as duration of green phase was fixed and speed limit was $50 \mathrm{~km} / \mathrm{h}$. Since the slope of a road has an influence on saturation flow and on start-up delay ([10]), it has been ensured to select only intersections without slope. Data of that study ([9]) originate from manually annotated video recordings, where snowy road condition have been observed on three different days. The green phase has been divided into equal measuring intervals, and the number of vehicles passing the stop line within one measuring interval has been determined. Saturation flow rate and start up delay have been estimated, applying a linear regression model as described in [12].

In the pre-study ([9]), effect of snowfall intensity has also been investigated, but it turned out that there was no influence on saturation flow or start-up delay. This is reasonable, since snowfall reduces visibility, which plays a minor role when speeds are as low as in urban areas. From video recordings and observations, the situation has been classified into fully covered with snow or no snow at all. In this respect, intensity or amount of snowfall are not important.

The target values are listed in Tables 1 and 2. It can be observed that saturation flow rate is different between all three locations during dry road conditions, whereas during snowy road conditions the measured values are almost equal. This indicates that a snowy road has a limiting effect on saturation flow rather than a relative reduction effect as proposed e.g. in [13]. Thus in this work the model calibration has not been performed on target values relative to normal (or dry road) conditions but exactly on the observed values for saturation flow and start up delay as listed in Tables 1 and 2 .

Table 1: Saturation flow rate of through lane in vphgpl and standard deviation (in brackets) as determined in $[9]$

\begin{tabular}{|l||c|c|}
\hline & Snowy Road & Dry Condition \\
\hline \hline Intersection 1 & $1215 / 20$ & $1821 / 32$ \\
\hline Intersection 2 & $1233 / 20$ & $1640 / 30$ \\
\hline Intersection 3 & $1255 / 15$ & $1565 / 32$ \\
\hline
\end{tabular}


Table 2: Start up delay at through lane in seconds and standard deviation (in brackets) as determined in [9]

\begin{tabular}{|l||c|c|}
\hline & Snowy Road & Dry Condition \\
\hline \hline Intersection 1 & $2.32 / 0.2$ & $2.29 / 0.15$ \\
\hline Intersection 2 & $1.96 / 0.19$ & $1.59 / 0.19$ \\
\hline Intersection 3 & $2.02 / 0.16$ & $2.17 / 0.21$ \\
\hline
\end{tabular}

\subsection{Selection of Parameters}

VISSIM is a very detailed microscopic model, based on psycho-physical parameters describing the perception of drivers (e.g. perceived speed differences in relation to space headway). As for other microscopic models, one part of the model describes car-following behaviour, whereas the other part describes lane changing. As the current study focuses on urban intersections, car following has been regarded as the dominating regime. Therefore the Wiedemann 74 model [14] has been used, which is recommended for urban roads. The main parameters for describing car following in the Wiedemann 74 model are desired speed, desired acceleration, desired deceleration and minimum following distance of vehicles. Lane changing behaviour has not been analysed, because it would require more detailed measurement data (e.g. trajectories from video data), which were not available for analysis.

The parameters of the car following model can be influenced during snowy road conditions for different reasons:

- Desired speed: Drivers are reducing their desired speed in order to avoid skidding. A reduction of desired speed, which reaches up to $30 \%$ for snowy roads, has been found by Perrin [15]. The reaction to adverse weather conditions varies between regions, probably because drivers are accustomed to different conditions as e.g. rainfall frequency.

- Desired acceleration / deceleration: Obviously a slippery road reduces friction between road surface and tires, thus drivers are not able to accelerate and decelerate as strong as compared to dry road conditions, i.e. maximum acceleration and deceleration decrease during snowy road conditions. Moreover drivers reduce acceleration and deceleration to avoid skidding.

- Minimum following distance: Drivers are trying to maintain a higher minimum following distance in order to cope with longer stopping distances caused by slippery roads. The resulting higher following distances can be measured as higher time headways at single vehicle detectors (see e.g. [2] on rainy conditions). 


\subsection{Simulation Outline}

For a single parameter setting one VISSIM simulation run has been performed for a duration of 1800 seconds using a random seed. The network consisted of a road section of $500 \mathrm{~m}$ with three lanes and one signalised intersection at the end of the road section. The traffic signal had a cycle length of 60 seconds with a green time of 32 seconds. All lanes were dedicated to through traffic. During the simulation vehicles have been individually detected at the stop line of intersection and the inflow of the network has been set to 7200 veh./h (approx. 2400 veh./h per lane), leading to an over saturated intersection within one signal cycle. Nevertheless measuring of vehicles at virtual detectors started after a warming-up period of two signal cycles in order to ensure an over saturated intersection. The green phase has been split into four measuring intervals (compare to [12]), leading to a sample size of 336 measurements for each parameter setting. Although only one simulation run has been performed for a given parameter setting, the measurements originate from different independent signal cycles. The saturation flow rate of the traffic model has been calculated in the same was as for the target values in [9], by applying a linear regression model on simulated vehicle data $[12]$.

\subsection{Sensitivity analysis}

As a preparatory step, the effect of each parameter on the target values (saturation flow rate and start up delay) has been investigated. First the parameters have been varied one at a time (OAT) in order to identify how saturation flow rate and start up delay changes. Secondly a method for identifying the effect of single parameters on the model's response has been applied. The purpose of this step is to identify unnecessary parameters and to discard them before calibration.

\subsubsection{One at a Time Analysis}

In order to identify the influence of each parameter on saturation flow rate and start up delay, they are investigated separately. This is done by varying one parameter at a time, while keeping all other factors fixed to a default value suggested by VISSIM. Moreover for comparison purposes the green time has been changed in an additional simulation run from 32 to 40 seconds.

\subsubsection{Elementary Effect Method}

Several approaches for identifying the sensitivity of individual parameters are described by Campolongo [16]. A sensitivity analysis applied on a microscopic traffic flow model has been carried out by Punzo [17]. In contrast to this work, Punzo determined the effect of parameters on the 
deviation between real vehicle trajectories and those determined by the traffic model. In both studies a method called "elementary effect" has been applied (see Formula 1).

$$
\begin{gathered}
E E_{i}=\frac{Y\left(x_{1}, \ldots, x_{i}+\Delta_{i}, \ldots, x_{k}\right)-Y\left(x_{1}, \ldots, x_{k}\right)}{\Delta_{i}} \\
E E_{i}=\frac{\sum_{i=1}^{r}\left|E E_{i}\right|}{r}
\end{gathered}
$$

$Y\left(x_{1}, \ldots, x_{k}\right)$ is the model's response (saturation flow rate and start up delay), where $x_{1}, \ldots, x_{k}$ are the $k$ parameters of the model. In Formula 1 only one parameter at a time is changed by $\Delta_{i}$ and the difference in the model's response before and after the change is calculated. This difference is related to step size $\Delta_{i}$. The method is based on a sample of $r$ trajectories where along one trajectory each parameter is increased or decreased by same the step $\Delta$. Thus each trajectory requires $(\mathrm{k}+1)$ simulation runs. As a prerequisite to apply this method each parameter has to be scaled to $[0,1]$. Finally the average over all trajectories for each parameter is calculated (see Formula 2). As mentioned in [16], to avoid effects of opposite signs due to non-monotonic behaviour of the model in Formula 2 the absolute value of $E E_{i}$ is averaged. A high elementary effect for any parameter indicates a high influence on the model's response and therefore this parameter has to be estimated more precisely. On the other hand parameters with a low elementary effect may be discarded from the calibration procedure.

\subsubsection{Calibration}

If all possible parameters of the car following model are changed during calibration the search space would become too large, and not all combinations can be evaluated in acceptable time. Therefore several parameters have been pre-selected and value ranges for the selected parameters have been chosen. Then the simulation has been run for all possible combinations (brute force calibration). Investigating all possible combination ensures a global optimum of calibration. Moreover not one single possible parameter setting has been expected, but rather a feasible region in the search space describing parameters settings resulting in correct target values of saturation flow rate and start up delay (as proposed in Tables 1 and 2). Another possibility is to apply random search methods that limits the search space by taking random or semi-random samples from the possible combinations of parameter values. 


\section{Calibration Results}

The results presented in this section refer to the steps described in the method section. First parameters are investigated independently, meaning that only one is changed while others are kept constant (OAT). Secondly the elementary effects of all car following parameters are estimated and visualised. This sensitivity analysis indicates whether a parameter has an effect and how the target value is influenced (e.g. in a linear or non-linear way).

\subsection{One at a Time Analysis - Results}

Following parameters have been varied one at a time while all other factors were fixed to default values:

- Deceleration: Desired deceleration is how intensive drivers are willing to break, whereas maximal possible deceleration is determined by physical limits i.e. the friction between road surface and tire. The maximal desired deceleration is of course limited by the maximal possible deceleration. Thus the desired deceleration has been changed systematically, but only negligible changes in saturation flow rate and start up delay could be found for this parameter. The effect of desired deceleration for different parameter settings has been investigated using the elementray effect method.

- Acceleration: In the same way as for deceleration, a desired and a maximal possible acceleration can be adjusted, but only the desired acceleration has been changed. The value for this parameter is additionally dependent on the prevailing speed, i.e. for moving vehicles a lower acceleration is expected than for vehicles starting from the stop line. In VISSIM by default a value of $3.5 \frac{\mathrm{m}}{\mathrm{sec}^{2}}$ at speeds between 0 and $10 \mathrm{~km} / \mathrm{h}$ is adjusted. Starting from these default values the desired acceleration has been reduced for each speed level. E.g. 100\% means no changes to default values, whereas $50 \%$ of desired acceleration resulted $1.75 \frac{\mathrm{m}}{\mathrm{sec}^{2}}$ for speeds between 0 and $10 \mathrm{~km} / \mathrm{h}$.

[Figure 1 about here.]

As visible in Figure 1 saturation flow rate decreases as desired acceleration is reduced. Start up delay tends to decrease as acceleration increases. The oscillation as visible in Figure 1(b) is an artifact of the applied regression model for estimating the start up delay. The start up delay is dependent on the number of vehicles in the first eight seconds after signal has switched to green, related to the total number of vehicles passing the intersection during the whole green phase. Since the number of vehicles within a time interval is an integer, this value 
may be constant, although acceleration has been decreased. On the other hand, the number of vehicles passing during the whole green phase also varies for small changes of acceleration. Thus it may happen that counts for the first interval are constant, while counts for the whole green phase are decreasing, which leads to the assumption that start up delay is increasing while acceleration is decreasing. Conversely the number of vehicles in the first interval may suddenly rise to a higher integer value, although acceleration has been varied only little. This leads to an abrupt change of start up delay, e.g. visible at $20 \%$ in Figure 1(b). Despite this, a general trend of an increasing start up delay with decreasing acceleration is visible. This is reasonable, since vehicles need more time for moving away from the intersection when acceleration is low.

- Desired Speed: The desired speed of a single vehicle is selected from a predefined probability distribution, which can be defined for each vehicle class. A uniform destribution between a minimum and maximum value is the default setting, but it is possible to define any shape for the distribution. In our work the mean value and its range have been varied, whereas always a uniform distribution has been assumed. Because in the model passenger cars have been simulated, only one speed distribution was required and had to be adjusted. In reality there are different classes of passenger cars. Heavy duty vehicles have different characteristics compared to passenger cars, and also the drivers of such cars might have different characteristics. This might come out in different preferences for speed and acceleration. However, the traffic characteristics that are targeted in this calibration are just the saturation flow and start up delay. Enlarging the number of parameters for the calibration will just make the result of the calibration less well determined: the more parameters are included to match with the value of only two output parameters, the more good solutions will exist. Therefore, the model to be calibrated is kept as simple as possible, while still remaining realistic.

[Figure 2 about here.]

In Figure 2 an influence of the desired speed (mean value) on saturation flow rate is visible. As desired speed decreases, the saturation flow rate decreases as well. The start up delay is changing less for higher speed values, but decreases down to $0.5 \mathrm{sec}$ for very low values (below $20 \mathrm{~km} / \mathrm{h}$ ). The start-up delay is caused by vehicles in the front line of a queue which have to accelerate up to their desired speed. For a given acceleration, this means that the higher the free speed, the longer an acceleration phase will last and the higher the start-up delay will be. This is the reason for the very short start-up delays at lower desired speeds. Range for desired speed distribution has been changed as well, but the range exhibited no effect on saturation flow or start up delay. The lower importance of this parameters is also shown by 
results from the elementary effect method.

- Minimum Following Distance: In the applied car following model (Wiedemann 74) two parameters are used for calculating minimum following distance of moving vehicles, called $b x_{m u l}$ and $b x_{a d d}([14])$. It is determined by

$$
b=\left(b x_{a d d}+b x_{m u l} \times z\right) \times \sqrt{v}
$$

where $z$ is a normally distributed random variable with 0.5 mean and variance of 0.15 (default values in VISSIM). $v$ is the velocity of the following vehicle. The sum of $b$ and a standstill distance represents the desired following distance between two consecutive vehicles, which is a variable in space. While $b x_{a d d}$ is a constant factor, $b x_{m u l}$ determines the variation of minimum following distance among different drivers. It has to be noted that this representation of the minimum following distance is markedly different from legal requirements and recommendations. For calculating a safe following distance, this distance is usually increased proportional to the increase in braking distance (which is a quadratic function of vehicle speed). Higher values for both $\left(b x_{m u l}\right.$ and $\left.b x_{a d d}\right)$ result in higher following distances between vehicles. As visible in Figure 3 an increased following distance leads to a decreased saturation flow rate.

For the start up delay this relation is not visible, meaning that same start up delay can be observed for different following distances. However $b x_{m u l}$ and $b x_{a d d}$ may not be changed without considering the required start up delay. In order to reduce the number of parameters for the brute force calibration, $b x_{m u l}$ and $b x_{a d d}$ have been combined into a single parameter called $b x$, where $b x=b x_{m u l}=b x_{a d d}$. As indicated in Figure 3 a change of $b x$ results in changing $b x_{m u l}$ and $b x_{a d d}$ along the black line. It is obvious that by varying only parameter $b x$ all possible values for saturation flow rate and start up delay can be achieved.

[Figure 3 about here.]

\subsection{Elementary Effect Results}

In the same way as for the OAT analysis, the elementary effect method has been applied on following parameters of the car following model:

- Desired deceleration

- Desired acceleration

- Desired speed and the speed range

- Parameters for determining minimum following distance ( $b x_{a d d}$ and $\left.b x_{m u l}\right)$ 
Table 3: Ranges and step width of parameters used for elementary effect method

\begin{tabular}{|l||c|}
\hline Parameter name & Range \\
\hline \hline Desired Deceleration, $m / s^{2}$ & {$[-0.4,-4.0]$} \\
\hline Desired Acceleration, $\%$ & {$[10,100]$} \\
\hline Desired Speed (Mean value), $\mathrm{km} / \mathrm{h}$ & {$[12.5,57.5]$} \\
\hline Desired Speed (Range), $\mathrm{km} / \mathrm{h}$ & {$[1,10]$} \\
\hline Minimum following distance $\left(b x_{a d d}\right)$ & {$[1,10]$} \\
\hline Minimum following distance $\left(b x_{m u l}\right)$ & {$[1,10]$} \\
\hline
\end{tabular}

All parameters have been varied within the ranges as listed in Table 3, by defining 10 equally distributed levels for each interval. For the range of the desired speed (uniform) distribution the value determines the difference between the two extreme values. In Figure 4 the elementary effects of parameters of the car following model are visualised for several simulation runs or trajectories respectively. As can been seen, average value becomes stable after selecting approximately 500 random trajectories.

Results show that acceleration, desired speed and following distance $\left(b x_{a d d}\right.$ and $\left.b x_{m u l}\right)$ exhibit considerable effects on saturation flow and start up delay. The effects of deceleration and desired speed range were much smaller.

[Figure 4 about here.]

Already during the OAT analysis, changes of deceleration exhibited a negligible influence on the model response. The elementary method confirms the very low effect on both saturation flow rate and start up delay. Furthermore, range of desired speed exhibits a very low effect on the model response as well. Therefore these two parameters has been discarded from calibration and set to a constant value.

\subsection{Calibration Results}

From the analysis, three remaining parameters resulted for calibration: acceleration, desired speed and combined parameter $b x$. In Table 4 the applied value ranges and number of levels are summarised. For calibration, a simulation run has been performed for all different possible combinations of parameters settings, $19 \times 9 \times 24=4104$. One run took about 30 seconds for a traffic simulation of 1800 seconds. The brute force method for calibration took about 34 hours on a common desktop PC, respresenting a feasible effort for calibration of microscopic simulation model.

The visualisation of saturation flow rate or start up delay in dependence of the three parameters at one glance is not possible. Therefore those simulation results have been selected where the saturation flow rate was within 1175 and 1285 vphgpl and start up delay between 1.58 and 2.72 
Table 4: Parameter and Levels

\begin{tabular}{|l||c|c|c|}
\hline Name & Min & Max & Levels \\
\hline \hline Acceleration, \% & 10 & 100 & 19 \\
\hline Desired Speed, km/h & 15 & 55 & 9 \\
\hline Min. Following Distance $(b x),(m \times s)^{0.5}$ & 0.25 & 6 & 24 \\
\hline
\end{tabular}

seconds, which is the minimum/maximum values measured at the three intersections investigated in [9], minus/plus two times the standard deviation (compare Table 1 and 2). Assuming a normal distribution for saturation flow and start up delay approximately $95 \%$ of all values would fall within this range. These target values are only valid for through lanes of arterial intersections without slope.

The result is a feasible region for possible parameter combinations to achieve a saturation flow rate and start up delay as measured during snowy road conditions (see Figure 5). For better visualisation the result of Figure 5 has been split up according to desired speed. In Figures ?? - 6(d) again a feasible region is plotted, where each plot refers to one desired speed value (see title of the figures). It is obvious that for a speed value between 30 and $50 \mathrm{~km} / \mathrm{h}$ the feasible regions are very similar. If a reduction within this range is expected (e.g. compare to Perrin [6]), the number of possible parameter settings can be decreased further. Further measurements with GPS trackers have been conducted in the road network of Vienna. Results show that acceleration ranged up to $2.5 \frac{\mathrm{m}}{\mathrm{s}^{2}}$ (99th percentile of all measured accelerations) for dry road conditions. In our case this represents a reduction to $71 \%$ of original acceleration settings. Taking into account these measurement results, the feasible region can be further limited.

[Figure 5 about here.]

[Figure 6 about here.]

\section{Conclusions and Future Work}

By sensitivity analysis three parameters have been identified as important parameters in order to calibrate a microscopic traffic model for snowy road conditions. Target values are saturation flow rate and start up delay which originate from previous investigations, where measurements on through lanes of urban intersections without slope have been considered. These values are similar for snowy road conditions rather than reduced values relative to values for dry weather conditions [9]. The sensitivity analysis indicated a negligible influence of desired deceleration and range of desired speed distribution. Furthermore parameters for calculating the minimum following 
distance could be combined to a single one. With the remaining parameters desired speed, desired acceleration and a combined parameter for the minimum following distance a brute force search method has been applied, and saturation flow rate and start up delay have been estimated from each simulation run. Finally the region in search space has been identified representing best fit to target values. This feasible region, which is a subspace of the search space, helps to identify parameters of VISSIM in order to achieve saturation flow rate for snowy road conditions. Furthermore saturation flow rate can be used in combination with information about signal timings to identify the capacity of signalised intersection. Although search space has been limited by the feasible region, several parameter settings are valid. However, the limited search space can provide more plausible initial values for an automatic calibration. With the help of already established calibration procedures (see i.a. [4]), VISSIM or any other microscopic traffic simulator can be calibrated to snowy road conditions. In this way, ITS applications as e.g. signal timing optimisation can be correctly planned or evaluated on urban roads on wintery road conditions.

In order to arrive at a more realistic representation of drivers' behaviour, additional investigations of traffic variables are required. By including findings from Perrin [15] concerning the reduction of desired speed, this region could be further reduced. Conducting further investigations on desired acceleration (within the defined limits) and minimum following distance during snowy road conditions would result in unambiguous values for model calibration. This might be done by a detailed analysis of vehicle trajectories, e.g. from video observations over a longer road section. As the preparation of trajectory data from video data is a very costly process, measurements of time headways at local detectors or driving cycles recorded with GPS tracking devices can be used as additional information for calibration [8]. For a complete simulation of traffic at intersections, reduction of saturation flow rates on turning lanes due to adverse weather conditions has to be investigated.

\section{REFERENCES}

[1] Maze TH, Agarwal M, Burchett G. Whether Weather Matters to Traffic Demand, Traffic Safety and Traffic Flow. Center for Transportation Research and Education, Iowa State University, USA; 2005.

[2] Billot R, Faouzi NEE, Vuyst FD. Multi-level Assessment of the Rain Impact on Drivers Behaviours: Standardized Methodology and Empirical Analysis. Transportation Research Records: Journal of the Transportation Research Board. 2009;2107(1):134-142.

[3] Bijleveld F, Churchill T. The Influence of Weather Conditions on Road Safety Research. SWOV Institue for Road Safety Research, Leidschendam, The Netherlands; 2009. 
[4] Sterzin ED. Modeling Influencing Factors in a Microscopic Traffic Simulator. Department of Civil and Environmental Engineering, Massachusetts Institute of Technology, Cambridge (USA); 2004.

[5] Chung E, Ohtani O, Warita H, Kuwahara M, Morita H. Does Weather Affect Highway Capacity? Institute of Industrial Science, University of Tokyo, Japan; 2006.

[6] Zhang L, Holm P, Colyar J. Identifying and Assessing Key Weather-Related Parameters and their Impacts on Traffic Operations Using Simulation. U.S. Department of Transportation, Colorado Springs; 2004.

[7] Nigarnjanagool S, Dia H. Evaluation of a Dynamic Signal Optimisation Control Model Using Traffic Simulation. IATSS Res (Int Assoc Traffic Saf Sci). 2005;29(1):22-30.

[8] Hirschmann K, Zallinger M, Fellendorf M, Hausberger S. A New Method to Calculate Emissions with Simulated Traffic Conditions. In: 13th International IEEE Annual Conference on Intelligent Transportation Systems, Madeira Island, Portugal; 2010. .

[9] Asamer J, vanZuylen HJ. Saturation Flow Under Adverse Weather Conditions. Transportation Research Record: Journal of the Transportation Research Board. 2011;2258:103-109.

[10] Transportation Research Board (TRB) Highway Capacity Manual, Special Report 209. National Research Council, TRB, Washington, D.C.; 2000.

[11] Wang MH, Benekohal RF. Analysis of Left-Turn Saturation Flow Rates and Capacity at Signalized Intersections. In: Proceedings of the 89th Annual TRB Meeting, Washington; 2010.

[12] Branston D, van Zuylen HJ. The Estimation of Saturation Flow, Effective Green Time and Passenger Car Equivalents at Traffic Signals by Multiple Linear Regression. Transportation Research Record: Journal of the Transportation Research Board. 1978;12(1):47-53.

[13] Botha JL, Kruse TR. Flow Rates at Signalized Intersections Under Cold Winter Conditions. Journal of Transportation Engineering. 1992;118(3):439-450.

[14] Wiedemann R. Simulation des Straßenverkehrsflusses. Schriftenreihe des IfV - Institut fr Verkehrswesen, University Karlsruhe (In German language). 1974;8.

[15] Perrin J, Martin PT, Hansen BG. Modifying Signal Timing During Inclement Weather. Transportation Research Record: Journal of the Transportation Research Board. 2001;1748:66-71.

[16] Campolongo F, Saltelli A, Cariboni J. From Screening to Quantitative Sensitivity Analysis. A Unified Approach. Computer Physics Communications. 2011;182:978-988.

[17] Punzo V, Ciuffo BF. Sensitivity Analysis of Microscopic Traffic Flow Models: Methodology and Application. In: Proceedings of the 90th Annual TRB Meeting, Washington; 2011. . 


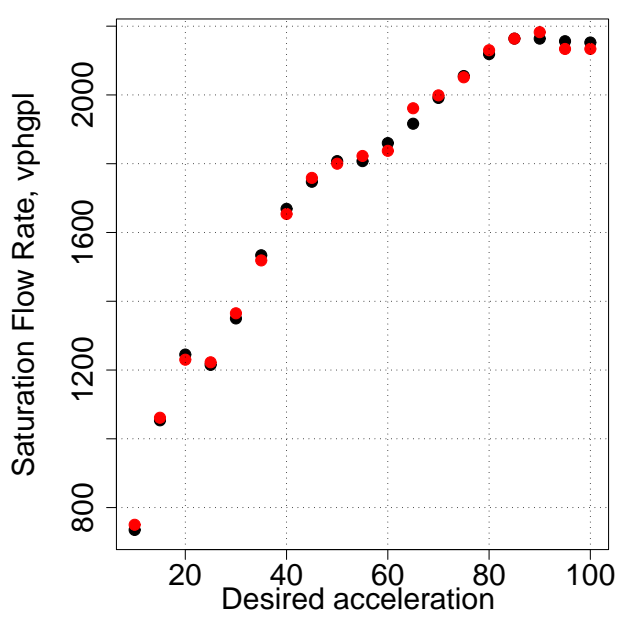

(Percentage of original value), \%

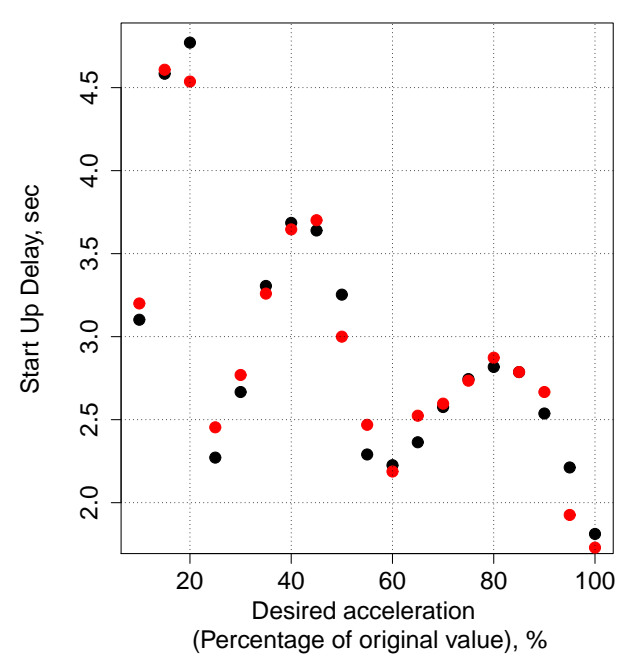

(b)

Fig. 1: Saturation flow rate (a) and start up delay (b) in dependence of acceleration for 32 sec (red)and $40 \mathrm{sec}$ (black) green time 


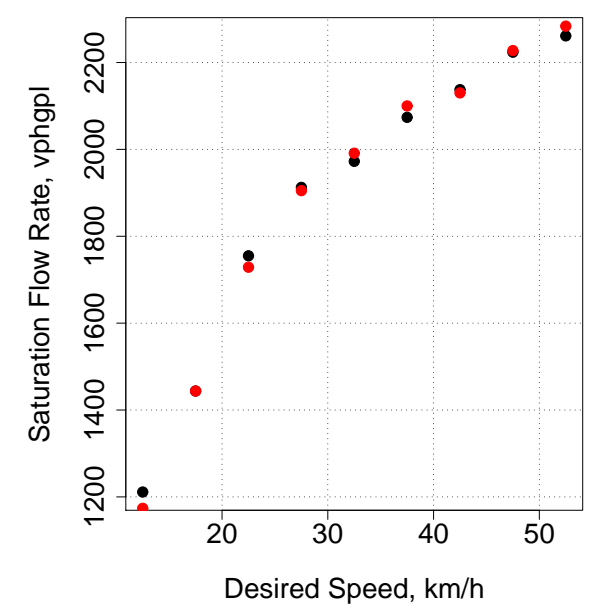

(a)

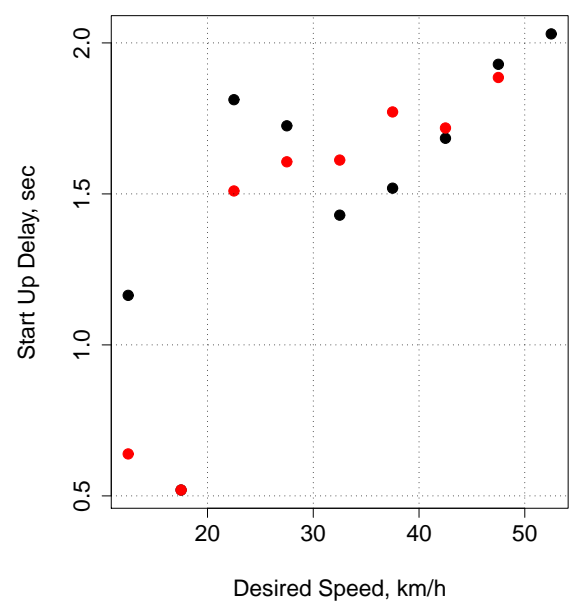

(b)

Fig. 2: Saturation flow (a) rate and start up delay (b) in dependence of desired speed (mean value) for $32 \mathrm{sec}$ (red)and $40 \mathrm{sec}$ (black) green time 


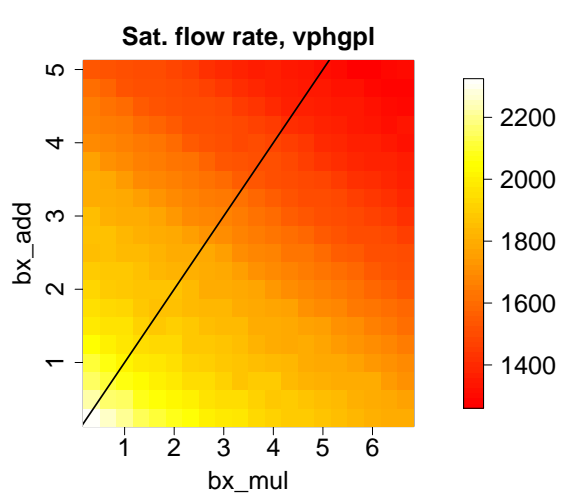

(a)

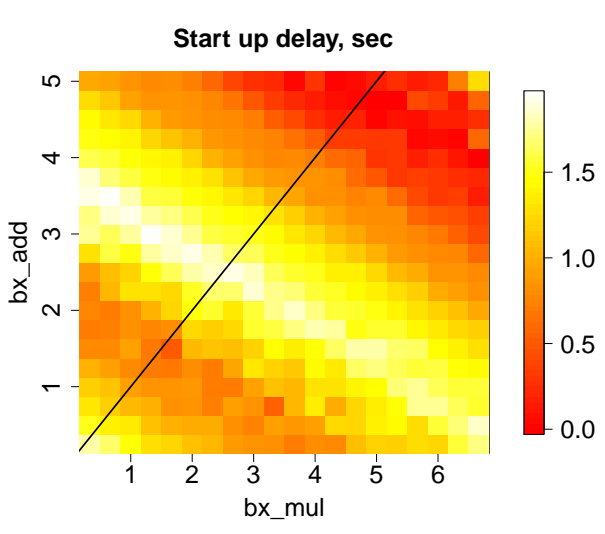

(b)

Fig. 3: Saturation flow rate (a) and start up delay (b) in dependence of $b x_{a d d}$ and $b x_{m u l}$. Black line indicates combined parameters $b x$ 


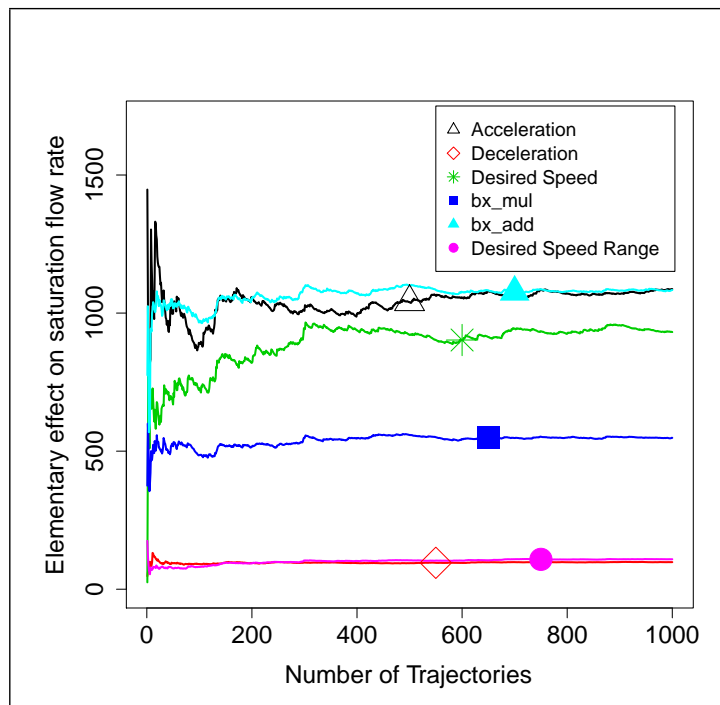

(a)

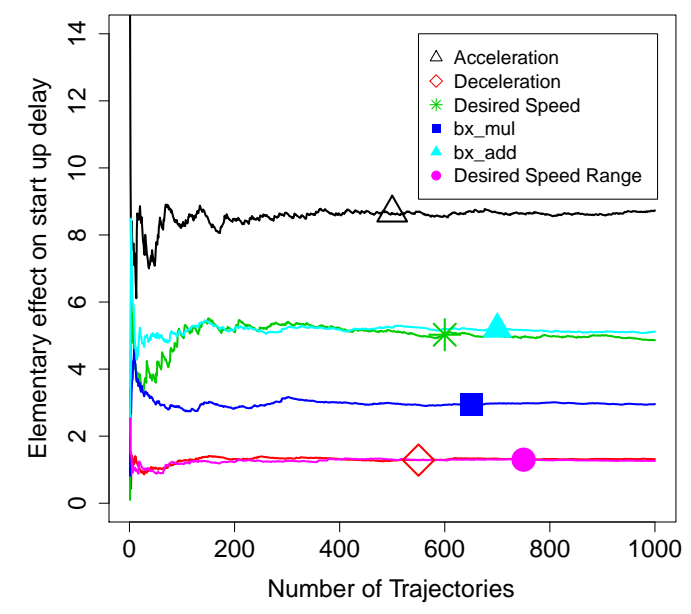

(b)

Fig. 4: Elementary effect on saturation flow rate (a) and start up delay (b) for several parameters 


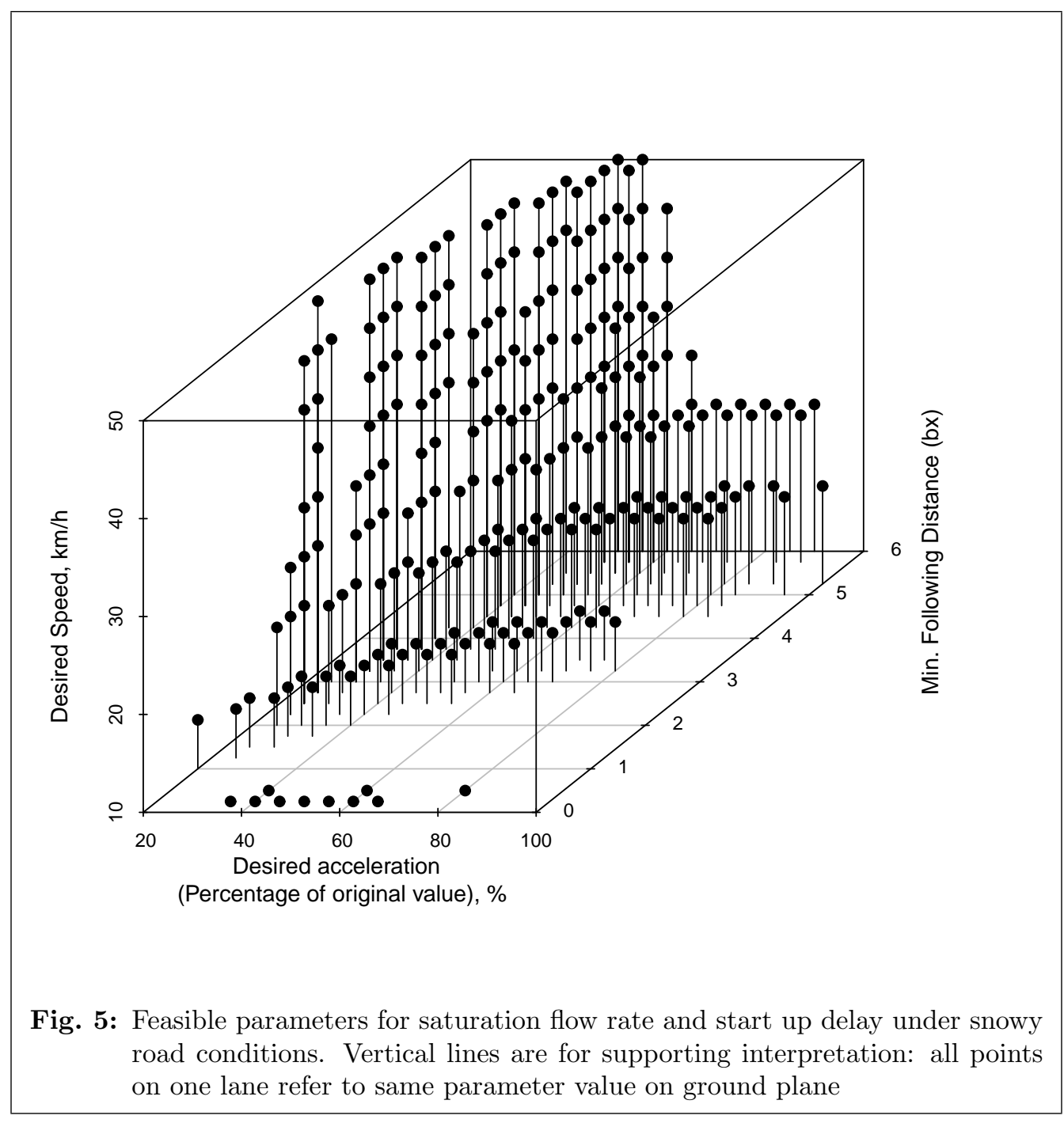




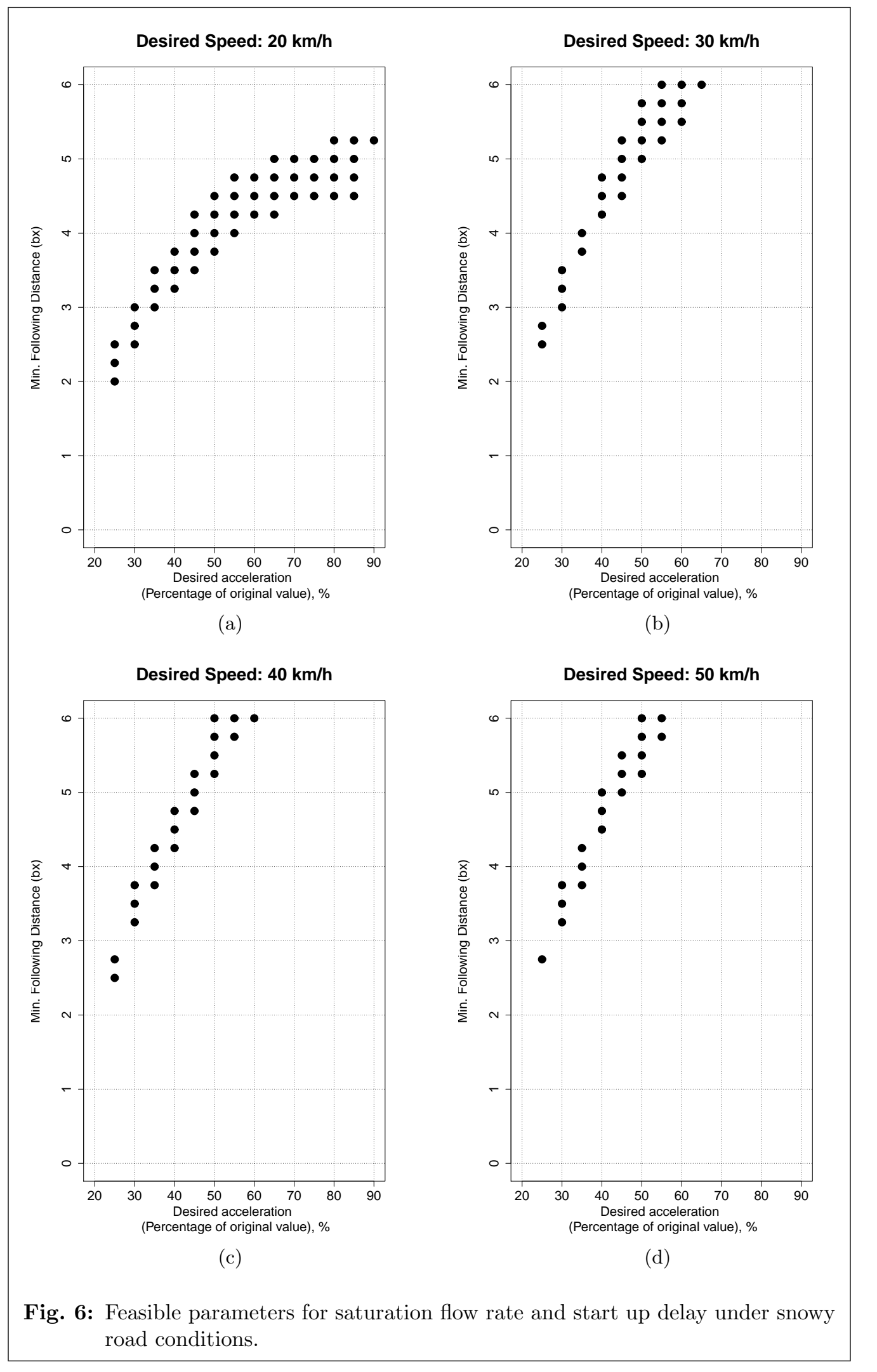

